\title{
Phenotypic expansion of autosomal dominant retinitis pigmentosa associated with the D477G mutation in RPE65
}

\author{
Ruben Jauregui, ${ }^{1,2,3}$ Ahra Cho, ${ }^{1,2,4}$ Jin Kyun Oh, ${ }^{1,2,5}$ Akemi J. Tanaka, ${ }^{6}$ \\ Janet R. Sparrow, ${ }^{1,2,6}$ and Stephen H. Tsang ${ }^{1,2,6}$ \\ ${ }^{1}$ Edward S. Harkness Eye Institute, Columbia University Medical Center, New York, New York 10032, USA; \\ ${ }^{2}$ Jonas Children's Vision Care and Bernard and Shirlee Brown Glaucoma Laboratory, New York, New York \\ 10032, USA; ${ }^{3}$ Weill Cornell Medical College, New York, New York 10065, USA; ${ }^{4}$ Institute of Human Nutrition, \\ Vagelos College of Physicians and Surgeons, Columbia University, New York, New York 10032, USA; ${ }^{5}$ State \\ University of New York at Downstate Medical Center, Brooklyn, New York 11203, USA; ${ }^{6}$ Department of \\ Pathology and Cell Biology, Columbia University Medical Center, New York, New York 10032, USA
}

Corresponding author: sht2@cumc.columbia.edu

(c) 2020 Jauregui et al. This article is distributed under the terms of the Creative Commons

Attribution-NonCommercial License, which permits reuse and redistribution, except for commercial purposes, provided that the original author and source are credited.

Ontology terms: congenital blindness; congenital visual impairment; decreased central vision

Published by Cold Spring Harbor Laboratory Press

doi:10.1101/mcs.a004952
Abstract Mutations in the gene RPE65 (OMIM: 180069) are recessively inherited and known to cause Leber congenital amaurosis. Recently, the mutation D477G in RPE65 has been identified as a cause of autosomal dominant retinitis pigmentosa (RP). Variable expressivity of this disease has been reported, as carrier individuals can present with mild, nonpenetrant, or, most commonly, a severe chorioretinal phenotype that resembles choroideremia. We report the case of a 57-yr-old male who presented to our clinic with nyctalopia and decreasing visual acuity for $1 \mathrm{yr}$. Dilated fundus examination revealed retinal atrophy and peripheral mottling of the retinal pigment epithelium (RPE). SW-AF revealed patchy hypoautofluorescence throughout the posterior pole with separate lacunae-like areas in the macula of severe RPE atrophy along with foveal sparing. Full-field electroretinogram suggested a rod-cone dystrophy. Whole-exome sequencing revealed the heterozygous mutation c. 1430A > G (p.D477G) in the RPE65 gene. This phenotype of peripheral RPE mottling and severe macular lacunae-like atrophy has not been previously reported with RPE65 autosomal dominant RP, supporting the variable expressivity of the disease and expanding the known phenotypic presentations.

\section{INTRODUCTION}

Retinitis pigmentosa (RP) refers to a group of retinal dystrophies that is a major cause of visual disability worldwide, with a prevalence of $\sim 1$ in 4000 for an estimated total of 1.5 million patients (Verbakel et al. 2018). The disease is caused in most cases by a primary degeneration of the rod photoreceptors followed by the cones, and thus is characterized as a rod-cone dystrophy (Hamel 2006; Hartong et al. 2006; Verbakel et al. 2018). The typical course of the disease for patients is initial nyctalopia, followed by progressive constriction of the visual fields and "tunneling" of vision. Macular function is preserved until late stages of the disease. On clinical examination, fundus abnormalities include intraretinal bone spicule pigment migration, attenuation of the retina vessels, and waxy pallor of the optic nerve. Disease severity generally depends on the Mendelian mode of inheritance: X-linked RP (5\%-15\% of patients) has a more severe course of disease as compared to autosomal recessive RP (50\%-60\%), 
whereas autosomal dominant RP $(30 \%-40 \%)$ is generally the mildest (Hamel 2006; Hartong et al. 2006; Verbakel et al. 2018; Jauregui et al. 2019b).

Leber congenital amaurosis (LCA) refers to a group of rod-cone dystrophies that present congenitally or within the first months of life with severe vision loss, nystagmus, and extinguished electroretinogram responses in most cases (Kumaran et al. 2017; Jauregui et al. 2018a; Sheck et al. 2018). LCA is inherited in an autosomal recessive manner, and $\sim 5 \%-$ $10 \%$ of cases are caused by pathogenic variants in the gene retinal pigment epithelium (RPE)-specific protein $65 \mathrm{kDa}$ (RPE65, OMIM: 180069) (Kumaran et al. 2017). This gene encodes for a retinol isomerase that serves as a vital component of the visual cycle by regenerating 11-cis-retinol from all-trans-retinyl ester (Shin et al. 2017). Recently, RPE65-LCA became the first retinal dystrophy to have Federal Drug Administration (FDA)-approved gene therapy after successful results from safety and efficacy studies (Russell et al. 2017).

In 2011, Bowne et al. (2011) reported the first study of the heterozygous RPE65 p.D477G variant causing autosomal dominant RP (adRP) in two Irish families. Further reports have shown that RPE65-adRP can present with chorioretinal atrophy resembling choroideremia (Bowne et al. 2011; Hull et al. 2016; Jauregui et al. 2018b). Nonpenetrance and mild disease have also been reported (Bowne et al. 2011; Hull et al. 2016). In this study, we describe a patient with the variant p.D477G in RPE65 who presented with a novel phenotype that has not been previously associated with this variant.

\section{RESULTS}

A 57-yr-old male patient was referred to our office for a retinal evaluation. The patient reported a subjective decrease in visual acuity beginning $\sim 1 \mathrm{yr}$ earlier with objects appearing "blurry" as well as decreased vision in low-light environments. Medical and ocular history were otherwise noncontributory. The patient's mother has a diagnosis of age-related macular degeneration, but there is no family history of vision problems similar to those of the patient.

On examination, best-corrected visual acuity (BCVA) was 20/50 bilaterally. Dilated fundus examination revealed widespread mottling of the RPE in the periphery, with more delineated areas of RPE atrophy centrally but with foveal sparing (Fig. 1A,B). Short-wave autofluorescence (SW-AF) imaging supported the findings from fundus examination. Patchy hypoautofluorescence was observed throughout the posterior pole of both eyes. In the right eye, multiple areas of RPE atrophy were observed on the macula, whereas in the left eye, there was only one similar area (Fig. 1C,D). The fovea was spared bilaterally. Spectral-domain optical coherence tomography (SD-OCT) scans revealed that in the areas of RPE atrophy, the ellipsoid zone (EZ) line and the underlying RPE and Bruch's membrane layers had degenerated, leading to a collapse of the overlying retinal layers (Fig. 1E,F). Outer retinal tubulations were also appreciated in the areas of atrophy (Fig. 1E). The retinal layers were conserved in the fovea. On full-field electroretinography (ff-ERG), the scotopic rod-specific responses were extinguished. Maximal responses exhibited a- and b-wave amplitudes of $87 \mu \mathrm{V}$ and $74 \mu \mathrm{V}$ in the right and $106 \mu \mathrm{V}$ and $105 \mu \mathrm{V}$ in the left eye. Photopic $30-\mathrm{Hz}$ flicker amplitudes were diminished to $62 \mu \mathrm{V}$ on the right and $48 \mu \mathrm{V}$ on the left eye. Genetic testing via whole-exome sequencing (WES) revealed the variant c.1430A > G (p.D477G) in the gene RPE65. No other additional variants were found.

\section{DISCUSSION}

The severe chorioretinal phenotype of RPE65-adRP caused exclusively by the D477G variant was first described in two Irish families in which the disease exhibited intrafamilial phenotypic 

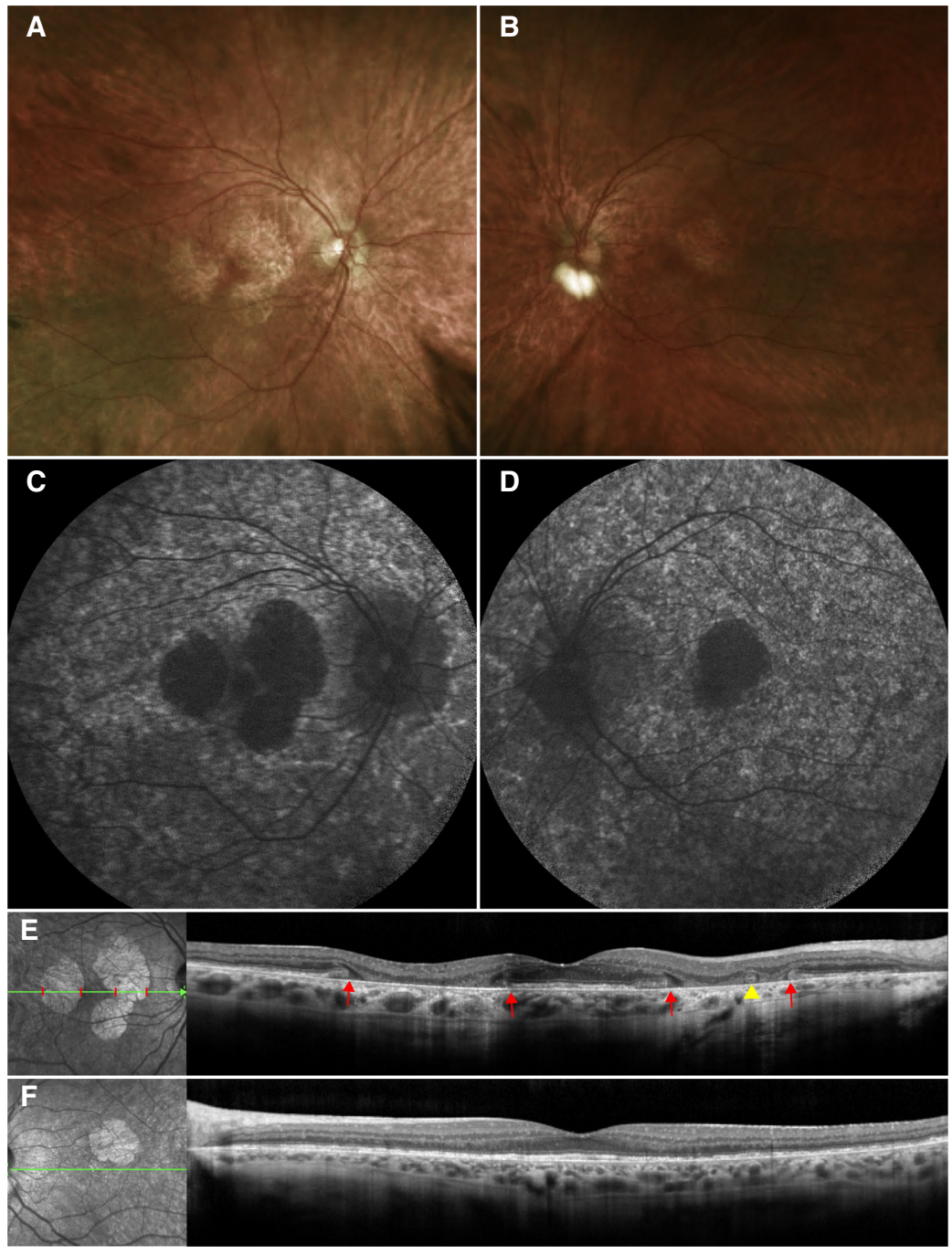

Figure 1. Clinical examination of the presented patient. Dilated fundus examination of the right eye $(A)$ revealed widespread mottling of the retinal pigment epithelium (RPE) in the periphery. More delineated areas of RPE atrophy are observed centrally with the underlying white sclera visible. RPE mottling and atrophy is also observed in the left eye (B) but to a lesser degree as compared to the right eye. There is sparing of the fovea bilaterally. Short-wavelength fundus autofluorescence of the right $(C)$ and left $(D)$ eyes revealed patchy hypoautofluorescence throughout the posterior pole. In the right eye, multiple areas of deep hypoautofluorescence corresponding to RPE atrophy were observed surrounding the fovea, whereas on the left eye there was only one such area. Spectral-domain optical coherence tomography (SD-OCT) scans and concurrently registered infrared images of the right $(E)$ and left $(F)$ eyes revealed that these lacunae-like, hypoautofluorescence areas correspond to regions where the ellipsoid zone (EZ) line and the underlying RPE and Bruch's membrane layers had degenerated, leading to a collapse of the overlying retinal layers (the boundaries are marked by red lines on the infrared image and by red arrows on the SD-OCT scan). Outer retinal tubulations are also appreciated (yellow arrowhead), suggesting photoreceptor and outer retinal degeneration. Outside of these areas of RPE atrophy, the retinal layers are intact.

variability (Bowne et al. 2011). In contrast to severe disease with extensive chorioretinal atrophy, nonpenetrance, and mild disease with peripheral pigment migration were also observed in some individuals (Bowne et al. 2011). Similarly, two different families in which both nonpenetrance and severe choroidal disease were observed have been reported 
(Hull et al. 2016). In addition, our group published a 2-yr follow-up study on a patient also afflicted with adRP caused by the same previously reported D477G variant in RPE65 (Jauregui et al. 2018b). Based on these studies, the severe chorioretinal phenotype associated with D477G RPE65-adRP can best be described as resembling choroideremia (Fig. 2). On fundoscopy, extensive chorioretinal atrophy is observed throughout the posterior pole, whereas in SW-AF images, there is extensive loss of autofluorescence in the areas of RPE atrophy, with scalloped regions of preserved retina and RPE. Unlike choroideremia, however, disease onset appears in adulthood for most patients with RPE65-adRP.

The patient we report in this study presents with a novel phenotype that has not been previously attributed to RPE65-adRP. In our patient, we observed RPE mottling by fundoscopy and patchy hypoautofluorescence in SW-AF imaging instead of severe chorioretinal atrophy of the posterior pole. Furthermore, our patient exhibits macular lacunae-like lesions of severe RPE atrophy with foveal sparing. In the areas of atrophy, outer retinal tubulations are appreciated, suggesting photoreceptor and outer retinal degeneration (Zweifel et al.
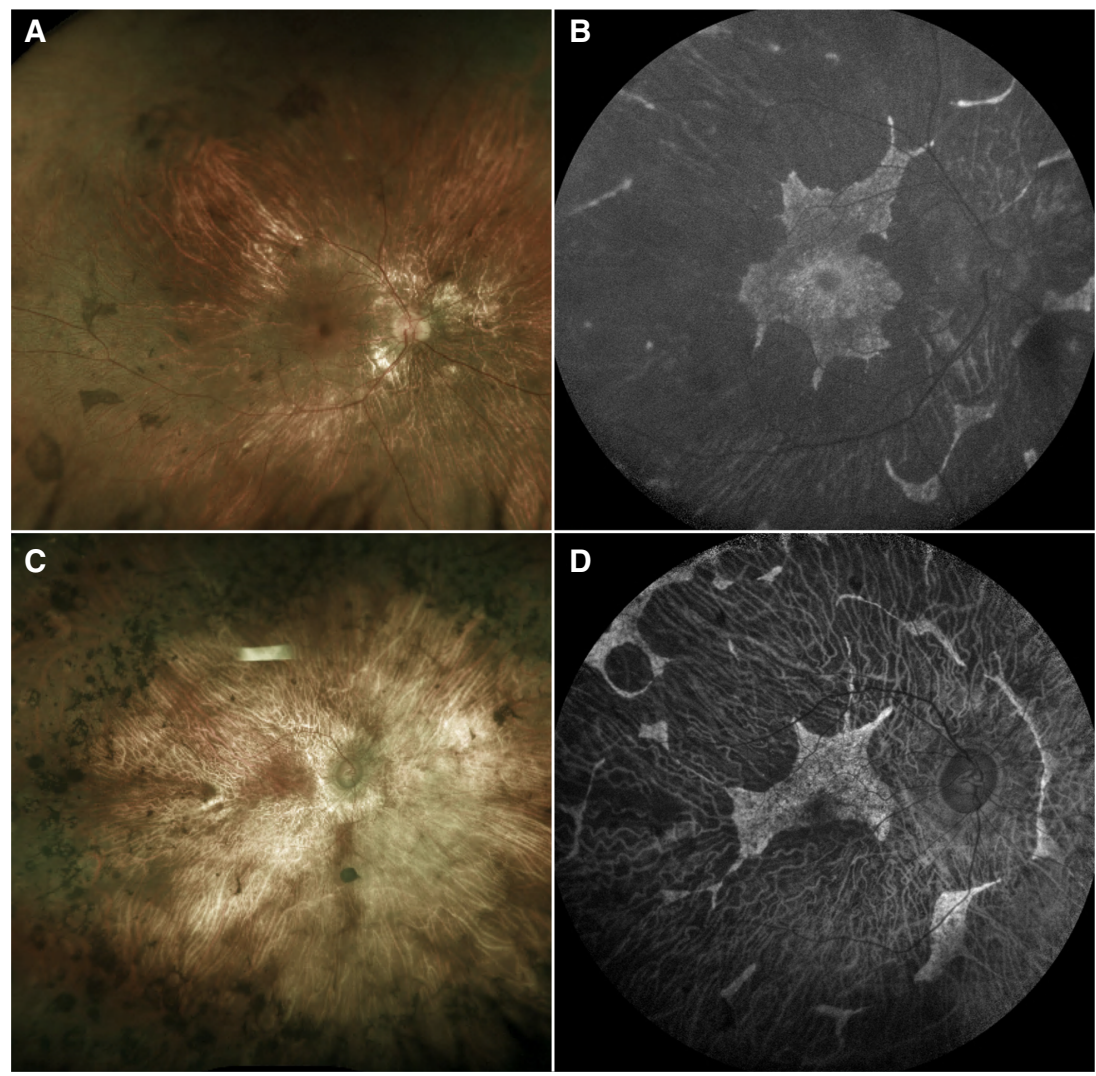

Figure 2. Clinical examinations of a patient with choroideremia and a patient with the classical phenotype of RPE65 autosomal dominant retinitis pigmentosa. Dilated fundus examination $(A)$ and short-wavelength fundus autofluorescence (SW-AF) imaging (B) of a 20-yr-old patient with choroideremia caused by the mutation c.1584_1587del (p.V529Hfs*7) in the gene CHM. Fundoscopy reveals chorioretinal atrophy, with the underlying white sclera visible on some areas of the posterior pole. On SW-AF, there is extensive loss of autofluorescence in the areas of RPE atrophy along with scalloped regions of preserved retina. The classically described phenotype of RPE65-autosomal dominant retinitis pigmentosa (adRP) resembles choroideremia. On dilated fundus examination $(C)$ and SW-AF imaging $(D)$ of a 69-yr-old patient with adRP caused by the mutation c.1430A > G (p.D477G) in RPE65, chorioretinal atrophy and scalloped regions of preserved retina in SW-AF images can be appreciated, thus resembling the phenotype observed in choroideremia. 
2009). Phenotypes similar to our patient's have previously been reported in female carriers of choroideremia, suggesting that RPE65-adRP can resemble either choroideremia in males or the carrier choroideremia state in females (Edwards et al. 2015; Jauregui et al. 2019a). In conjunction with previous reports, our study serves to illustrate that there is phenotypic variability of the D477G variant causing RPE65-adRP. Variable expressivity and incomplete penetrance are more common with adRP, and this phenomenon occurs in other genes that cause adRP such as PRPF8 and PRPF31 (Hamel 2006; Maubaret et al. 2011; Rose and Bhattacharya 2016).

The mechanism of pathogenicity of the D477G mutation has been explored by multiple studies. Initially, Bowne et al. (2011) proposed that the variant might destabilize folding of the tertiary RPE65 protein structure. A later study by Shin et al. used mutant knock-in mice and showed that D477G acts as a dominant negative mutation of RPE65 and causes a delay in the chromophore regeneration of the visual cycle (Shin et al. 2017). Choi et al. later demonstrated that the amino acid substitution is a toxic gain-of-function mutation, rather than a disturbance to protein folding or tertiary structure (Choi et al. 2018). Nevertheless, the most recent study on the mechanism of RPE65-adRP reported that D477G causes aberrant RNA splicing to create the pathogenic effect in humans and does not result in a gain-of-function mutation (Li et al. 2019). Similar to D477G RPE65-adRP, other genes that are known to cause variable expressivity and incomplete penetrance are also involved in RNA processing, such as PRPF8 and PRPF31 (Maubaret et al. 2011; Rose and Bhattacharya 2016). The variable expressivity of the D477G mutation, including nonpenetrance in some carriers, suggests that there might be additional mutations or modifier effects by other genes. Therefore, it is integral to perform further genomic analysis to help elucidate possible linkage of multiple genes involved in the pathogenicity of this mutation.

In conclusion, this study expands the currently known phenotypic presentations of adRP caused by the D477G mutation in RPE65. This study also serves to increase awareness of the variable phenotypic presentations of RPE65-retinopathy. In patients in which phenotypic presentation suggests choroideremia or a choroideremia carrier state but targeted sequencing of the gene CHM is negative, sequencing of RPE65 should be pursued.

\section{METHODS}

\section{Subjects and Ophthalmic Evaluation}

Ophthalmic evaluation of the patient included measurement of best corrected visual acuity, pupillary dilation $(>7 \mathrm{~mm}$ ) followed by fundus examination, photography, SD-OCT, and SW-AF (488 nm). ff-ERG was performed using DTL recording electrodes and Ganzfeld stimulation according to the standards from the International Society for Clinical Electrophysiology of Vision (ISCEV) (McCulloch et al. 2015a,b).

\section{DNA Analysis}

DNA was obtained from the patient's peripheral blood to perform clinical WES. WES was performed using SureSelectXT Human All Exon V5 + UTRs (Agilent Technologies) capture

\begin{tabular}{|c|c|c|c|c|c|c|c|}
\hline Gene & Chromosome & $\begin{array}{l}\text { HGVS DNA } \\
\text { reference }\end{array}$ & $\begin{array}{l}\text { HGVS protein } \\
\text { reference }\end{array}$ & $\begin{array}{l}\text { Variant } \\
\text { type }\end{array}$ & $\begin{array}{l}\text { Predicted } \\
\text { effect }\end{array}$ & $\begin{array}{l}\text { dbSNP/ } \\
\text { dbVar ID }\end{array}$ & Genotype \\
\hline RPE65 & $\begin{array}{c}\text { Chr 1:68896768T >C } \\
(\text { GRCh37/hg19) }\end{array}$ & c. $1430 A>G$ & p.Asp477Gly & Missense & Substitution & Not available & Heterozygous \\
\hline
\end{tabular}


Table 2. Whole-exome sequencing coverage table for the RPE65 c.1430A > G (p.Asp477Gly) variant

Percentage of RPE65 sites

\begin{tabular}{lccc} 
Sample & Percentage of reads aligned & Average read coverage & $\begin{array}{c}\text { Percentage of RPE65 sites } \\
(2605 \text { bases }) \text { with } \geq 10 \times \text { coverage }\end{array}$ \\
\hline Proband & $97.28 \%$ & 146.72 & 100
\end{tabular}

and HiSeq2500 (Illumina) sequencing technology. Sequence reads obtained were analyzed for the presence of pathogenic mutations by alignment to the human genome reference sequence (GRCh37/hg19) using the NextGENe software (Softgenetics) and our own analytical pipeline at the Laboratory of Personalized Genomic Medicine at Columbia University (Nagy and Mansukhani 2015). Identified variants were assessed for clinical phenotypic match and classified according to the American College of Medical Genetics and Genomics (ACMG) guidelines for the interpretation of sequence variants (Kalia et al. 2017). Variant information is provided in Table 1; sequencing coverage data is provided in Table 2.

\section{ADDITIONAL INFORMATION}

\section{Data Deposition and Access}

Whole-exome sequencing data is not publicly available as our patient consent does not permit the data to be uploaded to a depository. Patient consent could not be obtained. The NM_000329.3(RPE65):c.1430A > G (p.D477G) variant can be accessed through OMIM Allelic Variant 180069.0013.

\section{Ethics Statement}

This study is in accordance to the tenets from the Declaration of Helsinki and was approved by the Institutional Review Board (IRB) of Columbia University Medical Center. Informed consent was obtained from the patients as outlined by the IRB protocol \#AAAR0284. As per a retrospective study with no more than a minimal risk for the subject, this study did not require written consent by IRB policies.

\section{Acknowledgments}

Thanks and appreciation are extended to the patient described for the willingness to participate.

\section{Author Contributions}

R.J., J.R.S., and S.H.T. conceived the study design. R.J., A.C., A.J.T., and J.K.O. wrote the manuscript text. R.J. and A.C. gathered data and images for the study. J.R.S. and S.H.T. supervised the study and provided resources. All authors reviewed and approved the final version of the manuscript.

Competing Interest Statement

The authors have declared no competing interest.

Received November 11, 2019; accepted in revised form December 5, 2019.

\section{Funding}

The Jonas Children's Vision Care and Bernard \& Shirlee Brown Glaucoma Laboratory are supported by the National Institutes of Health (P30EY019007, R01EY018213, R01EY024698, R01EY024091, R01EY026682, R21AG050437), Foundation Fighting Blindness (TA-NMT-0116-0692-COLU), National Cancer Institute Core (5P30CA013696), and the Research to Prevent Blindness (RPB) Physician-Scientist Award. 


\section{REFERENCES}

Bowne SJ, Humphries MM, Sullivan LS, Kenna PF, Tam LC, Kiang AS, Campbell M, Weinstock GM, Koboldt DC, Ding L, et al. 2011. A dominant mutation in RPE65 identified by whole-exome sequencing causes retinitis pigmentosa with choroidal involvement. Eur J Hum Genet 19: 1074-1081. doi:10.1038/ejhg .2011 .86

Choi EH, Suh S, Sander CL, Hernandez CJO, Bulman ER, Khadka N, Dong Z, Shi W, Palczewski K, Kiser PD. 2018. Insights into the pathogenesis of dominant retinitis pigmentosa associated with a D477G mutation in RPE65. Hum Mol Genet 27: 2225-2243. doi:10.1093/hmg/ddy128

Edwards TL, Groppe M, Jolly JK, Downes SM, MacLaren RE. 2015. Correlation of retinal structure and function in choroideremia carriers. Ophthalmology 122: 1274-1276. doi:10.1016/j.ophtha.2014.12.036

Hamel C. 2006. Retinitis pigmentosa. Orphanet J Rare Dis 1: 40. doi:10.1186/1750-1172-1-40

Hartong DT, Berson EL, Dryja TP. 2006. Retinitis pigmentosa. Lancet 368: 1795-1809. doi:10.1016/S01406736(06)69740-7

Hull S, Mukherjee R, Holder GE, Moore AT, Webster AR. 2016. The clinical features of retinal disease due to a dominant mutation in RPE65. Mol Vis 22: 626-635.

Jauregui R, Cho GY, Takahashi VKL, Takiuti JT, Bassuk AG, Mahajan VB, Tsang SH. 2018a. Caring for hereditary childhood retinal blindness. Asia Pac J Ophthalmol (Phila) 7: 183-191. doi:10.22608/APO .201851

Jauregui R, Park KS, Tsang SH. 2018b. Two-year progression analysis of RPE65 autosomal dominant retinitis pigmentosa. Ophthalmic Genet 39: 544-549. doi:10.1080/13816810.2018.1484929

Jauregui R, Park KS, Tanaka AJ, Cho A, Paavo M, Zernant J, Francis JH, Allikmets R, Sparrow JR, Tsang SH. 2019a. Spectrum of disease severity and phenotype in choroideremia carriers. Am J Ophthalmol 207: 77-86. doi:10.1016/j.ajo.2019.06.002

Jauregui R, Takahashi VKL, Park KS, Cui X, Takiuti JT, Lima de Carvalho JR Jr, Tsang SH. 2019b. Multimodal structural disease progression of retinitis pigmentosa according to mode of inheritance. Sci Rep 9: 10712. doi:10.1038/s41598-019-47251-z

Kalia SS, Adelman K, Bale SJ, Chung WK, Eng C, Evans JP, Herman GE, Hufnagel SB, Klein TE, Korf BR, et al. 2017. Recommendations for reporting of secondary findings in clinical exome and genome sequencing, 2016 update (ACMG SF v2.0): a policy statement of the American College of Medical Genetics and Genomics. Genet Med 19: 249-255. doi:10.1038/gim.2016.190

Kumaran N, Moore AT, Weleber RG, Michaelides M. 2017. Leber congenital amaurosis/early-onset severe retinal dystrophy: clinical features, molecular genetics and therapeutic interventions. Br J Ophthalmol 101: 1147-1154. doi:10.1136/bjophthalmol-2016-309975

Li Y, Furhang R, Ray A, Duncan T, Soucy J, Mahdi R, Chaitankar V, Gieser L, Poliakov E, Qian H, et al. 2019. Aberrant RNA splicing is the major pathogenic effect in a knock-in mouse model of the dominantly inherited c.1430A>G human RPE65 mutation. Hum Mutat 40: 426-443. doi:10.1002/humu .23706

Maubaret CG, Vaclavik V, Mukhopadhyay R, Waseem NH, Churchill A, Holder GE, Moore AT, Bhattacharya SS, Webster AR. 2011. Autosomal dominant retinitis pigmentosa with intrafamilial variability and incomplete penetrance in two families carrying mutations in PRPF8. Invest Ophthalmol Vis Sci 52: 9304-9309. doi:10.1167/iovs.11-8372

McCulloch DL, Marmor MF, Brigell MG, Hamilton R, Holder GE, Tzekov R, Bach M. 2015a. Erratum to: ISCEV standard for full-field clinical electroretinography (2015 update). Doc Ophthalmol 131: 81-83. doi:10 .1007/s10633-015-9504-z

McCulloch DL, Marmor MF, Brigell MG, Hamilton R, Holder GE, Tzekov R, Bach M. 2015b. ISCEV Standard for full-field clinical electroretinography (2015 update). Doc Ophthalmol 130: 1-12. doi:10.1007/s10633-0149473-7

Nagy PL, Mansukhani M. 2015. The role of clinical genomic testing in diagnosis and discovery of pathogenic mutations. Expert Rev Mol Diagn 15: 1101-1105. doi:10.1586/14737159.2015.1071667

Rose AM, Bhattacharya SS. 2016. Variant haploinsufficiency and phenotypic non-penetrance in PRPF31-associated retinitis pigmentosa. Clin Genet 90: 118-126. doi:10.1111/cge.12758

Russell S, Bennett J, Wellman JA, Chung DC, Yu ZF, Tillman A, Wittes J, Pappas J, Elci O, McCague S, et al. 2017. Efficacy and safety of voretigene neparvovec (AAV2-hRPE65v2) in patients with RPE65-mediated inherited retinal dystrophy: a randomised, controlled, open-label, phase 3 trial. Lancet 390: 849-860. doi:10 .1016/S0140-6736(17)31868-8

Sheck L, Davies WIL, Moradi P, Robson AG, Kumaran N, Liasis AC, Webster AR, Moore AT, Michaelides M. 2018. Leber congenital amaurosis associated with mutations in CEP290, clinical phenotype, and natura history in preparation for trials of novel therapies. Ophthalmology 125: 894-903. doi:10.1016/j.ophtha 2017.12.013 
Shin Y, Moiseyev G, Chakraborty D, Ma JX. 2017. A dominant mutation in Rpe65, D477G, delays dark adaptation and disturbs the visual cycle in the mutant knock-in mice. Am J Pathol 187: 517-527. doi:10.1016/j .ajpath.2016.11.004

Verbakel SK, van Huet RAC, Boon CJF, den Hollander Al, Collin RWJ, Klaver CCW, Hoyng CB, Roepman R Klevering BJ. 2018. Non-syndromic retinitis pigmentosa. Prog Retin Eye Res 66: 157-186. doi:10.1016/j .preteyeres.2018.03.005

Zweifel SA, Engelbert M, Laud K, Margolis R, Spaide RF, Freund KB. 2009. Outer retinal tubulation: a novel optical coherence tomography finding. Arch Ophthalmol 127: 1596-1602. doi:10.1001/archophthalmol .2009 .326 


\section{COLD SPRING HARBOR Molecular Case Studies}

\section{Phenotypic expansion of autosomal dominant retinitis pigmentosa associated with the D477G mutation in RPE65}

Ruben Jauregui, Ahra Cho, Jin Kyun Oh, et al.

Cold Spring Harb Mol Case Stud 2020, 6: a004952

Access the most recent version at doi: $10.1101 / \mathrm{mcs} . a 004952$

References This article cites 23 articles, 2 of which can be accessed free at: http://molecularcasestudies.cshlp.org/content/6/1/a004952.full.html\#ref-list-1

License This article is distributed under the terms of the Creative Commons Attribution-NonCommercial License, which permits reuse and redistribution, except for commercial purposes, provided that the original author and source are credited.

Email Alerting Receive free email alerts when new articles cite this article - sign up in the box at the Service top right corner of the article or click here. 